\title{
Review Article \\ TNM-Like Classification: A New Proposed Method for Heart Failure Staging
}

\author{
Francesco Fedele, Maria Chiara Gatto, Alessandra D’Ambrosi, and Massimo Mancone \\ Department of Cardiovascular, Respiratory, Nephrology, Anesthesiology and Geriatric Sciences, Sapienza University of Rome, \\ Policlinico Umberto I, Viale del Policlinico 155, 00161 Rome, Italy
}

Correspondence should be addressed to Francesco Fedele; francesco.fedele@uniromal.it

Received 10 July 2013; Accepted 29 September 2013

Academic Editors: X. Ai and Y. Yang

Copyright ( 2013 Francesco Fedele et al. This is an open access article distributed under the Creative Commons Attribution License, which permits unrestricted use, distribution, and reproduction in any medium, provided the original work is properly cited.

\begin{abstract}
Heart Failure (HF) is an acute or chronic syndrome, that causes a lot of damaging effects to every system. The involvement of different systems is variably related to age and others comorbidities. The severity of organ damage is often proportional to the duration of heart failure. The typology of HF and the duration determine which organs will be affected and vice versa the severity of organ damage supplies precious information about prognosis and outcome of patients with heart failure. Moreover, a classification based not only on symptomatic and syndromic typical features of heart failure, but also on functional data of each system, could allow us to apply the most appropriate therapies, to obtain a more accurate prognosis, and to employ necessary and not redundant human and financial resources. With an eye on the TNM staging used in oncology, we drawn up a classification that will consider the different involvement of organs such as lungs, kidneys, and liver in addition to psychological pattern and quality of life in HF patients. For all these reasons, it is our intention to propose a valid and more specific classification available for the clinical staging of HF that takes into account pathophysiological and structural changes that can remark prognosis and management of HF.
\end{abstract}

\section{Introduction}

Heart Failure (HF) is an acute or chronic unhealthy condition, characterized by the inability of the heart to warrant its pump function in terms of adequate oxygen supply to the body tissues. An enchanting explanation of the HF's condition was proposed by Neubauer who defined HF like an "engine out of fuel" [1]. Even though the pump's failure is common to both acute and chronic HF, these differ from one another in HF's duration and the kind of organ damage developed. The prevalence of HF is greater in the population over 50 years old and the incidence is directly correlated with age [2]. The incidence of HF is steadily increasing because the ageing of the population, especially in the occidental world. Moreover, hospitalization and domiciliary treatments for HF represent an important health care burden [3]. The incidence of $\mathrm{HF}$ is destined to increase substantially over the next decade [4]. Recent updates of AHA/ACC guidelines about HF focus on diagnosis and management of HF in adults [5]. The current methods for clinical assessment of HF subdivide patients with $\mathrm{HF}$ or with high risk for $\mathrm{HF}$ in four classes or stages. In the first two stages (A and B), the patients are asymptomatic, whereas in the last two stages, they have a clinically manifested HF (stage C) which becomes refractory to therapy (stage D). The current guidelines modulate therapeutic interventions on the basis of this classification.

In 2006, Senni et al. [6] claimed that the predictor parameters identified by clinical studies and trials may be often unrepresentative of HF in the community. Senni in his study highlights the importance of prognostic stratification in patients with $\mathrm{HF}$ and proposes the use of Cardiovascular Medicine Heart Failure (CVM-HF) index as a valuable tool for the prognosis of stable HF.

The authors focused not only on cardiac parameters but also on comorbidity of patients with HF and developed a model to evaluate the 1-year risk mortality at all stages of the disease. The CVM-HF index includes 13 variables: age, anemia, hypertension, chronic obstructive pulmonary disease, complicated diabetes mellitus, moderate to severe kidney dysfunction, metastatic cancer, absence of $\beta$ blockers therapy, 
absence of angiotensin-converting enzyme inhibitors/angiotensin receptor-antagonist, NYHA (New York Heart Association) class III/IV, left ventricular ejection fraction $\leq 20 \%$, severe valvular heart disease, and atrial fibrillation. Depending on the score achieved, patients are considered in the low-, medium-, and high-risk group [6].

In 2009, Cygankiewicz et al. on Behalf of the MUSIC investigators, realized a study that proposed the evaluation of dynamic electrocardiographic measures to identify patients at risk of all-cause mortality and cardiac death [7].

In the same year, Vazquez et al. proposed the MUSIC Risk Score, a simple method that evaluates a limited number of variables tested with noninvasive methods. In the MUSIC Risk Score, demographic, clinical, echocardiographic, 12-lead ECG, and $24 \mathrm{~h}$ Holter monitoring and laboratory variables were taken into account to predict mortality in ambulatory patients with chronic HF [8].

Kalogeropoulos et al. in a recent study, tested the Seattle Heart Failure Model (SHFM) in patients with advanced HF. The study showed that this model overestimates survival, particularly in patients with implanted devices. On the contrary, in blacks, SHFM showed underestimation of the risk in patients with advanced HF [9].

Another risk score to assess in-hospital mortality in patients with $\mathrm{HF}$ was validated by Peterson et al. using American Heart Association Get With the Guidelines-Heart Failure (GWTG-HF) program data. This risk-score was established using variables identified in the multivariate model. The variables used were age, sex, race, atrial arrhythmia, diabetes, chronic obstructive pulmonary disease, peripheral vascular disease, coronary artery disease, cerebrovascular disease, ischemic etiology, depression, ejection fraction, blood urea nitrogen (BUN), sodium, hemoglobin, creatinine, heart rate, and systolic blood pressure [10].

More recently, Peterson et al. indicate a paradoxical inverse relationship between treatment and risk in HF. In practice, higher-risk patients are less likely to receive recommended therapy. This "mismatch treatment" is probably due to the fact that the patients with $\mathrm{HF}$ at high risk of mortality have more contraindications to drugs resulting in being ineligible to evidence-based therapy.

For all these reasons, one of the unmet needs in the field of HF is a clinical classification that accurately stratifies our patients in order to (a) make a more precise prognostic evaluation and (b) find the more appropriate treatment, not only in term of efficacy, but also in terms of cost-benefits [11]. A classification of this type, should enable the physician to better manage the patient's illness, but mainly the human and material resources available. In fact, a classification that takes into account comorbidities and their severity in patients with heart failure may also allow a different reimbursement of expenses incurred by national health systems and insurance. Only by framing the main organs involved in HF and their relationship, we can choose the most appropriate therapy to be adopted in a wide range of therapeutic tools available.

In this setting, we propose a staging system for $\mathrm{HF}$ similar to the TNM evaluation used in oncology [12]. The evaluation of myocardial damage is necessary, as well as the type of changes that can occur in the myocardial structure and function. This is the first step where " $\mathrm{H}$ "- for Heartmay be the analogue of " $\mathrm{T}$ " from the aforementioned TNM. The second step in this HF staging is the assessment of lungs involvement. For their functional and anatomical closeness to the heart, they may be indeed considered, continuing the analogy, as a lymph node station. Finally, remembering the etymological meaning of "metastasis," that in Ancient Greek signified "what is beyond there," similarly to the concept used in oncology for metastasization, the "Malfunction" of peripheral organs like kidney, liver, brain, and so forth may represent the final step "M."

\section{Materials and Methods}

2.1. " $H$ " for Heart. To evaluate the extension of heart impairment, we propose to consider not only systolic and diastolic functions, in terms of ejection fraction and transmitral flow, but also other characteristical anatomo-functional conditions, as previous myocardial infarction and cardiac remodeling (hypertrophy and or dilatation). Previous myocardial infarction and its extension is a fundamental parameter to be considered in this staging process. Echocardiography is a valid tool to be used for the evaluation of the functional consequences of the ischemic-necrotic process. However, more recently, the magnetic resonance imaging has proved to add further information on tissue characterization, and precisely, on transmural extension of the infarct and myocardial viability $[13,14]$. Left ventricular hypertrophy, well evaluable by echocardiography, is the consequence of a series of cardiac and vascular changes. Various etiologies, as hypertension or hypertrophic cardiomyopathies, account for left ventricular hypertrophy [15-17]. Left ventricular remodeling is easily evaluable by echocardiography, that allows the estimate of diameters (systolic and diastolic normal values: $50 \pm 5 \mathrm{~mm}$ and $31 \pm 5 \mathrm{~mm}$, resp. [18]) volumes and shapes. The worst condition of heart in $\mathrm{HF}$ is represented by biventricular dysfunction that involves both the left and the right ventricles.

All these aforementioned parameters have a consolidated prognostic value. Matching in different ways these parameters, grading them from less to more severe conditions, we can obtain a staging of cardiac damage during $\mathrm{HF}$ (from $\mathrm{H}_{1}$ to $\mathrm{H}_{4}$ : see scheme in Table 1 ).

2.2. " $L$ " for Lung. The evaluation of grading and timing of pulmonary system's damage is necessary to give awareness of progress and severity of HF. The clinical manifestations of lungs' implication in HF are multifaceted. In this meaning, the relationship between heart and lungs is to be considered biunique. Even though, in a first moment, left ventricular dysfunction leads to pulmonary congestion, which reveals itself the pulmonary hypertension [19] occurrence, in second time, pulmonary hypertension and pulmonary volume overload precipitate right ventricular dysfunction [20]. Numerous evidences establish that the presence of pressure increment in pulmonary vasculature predicts a poor outcome in patients with HF [21]. Pulmonary hypertension also occurs in patients with HF and preserved ejection fraction [22]. Therefore, considering pulmonary involvement only in patients with 
TABLE 1: (a) The parameters considered in the staging of the heart damage. (b) See text for the explanation (LV: left ventricle).

(a)

\begin{tabular}{l}
\hline Cardiac damages \\
\hline Hypertrophy \\
Transmitral flow \\
Previous N-STEMI \\
Previous STEMI \\
Ventricular remodeling \\
Ejection fraction $<35 \%$ \\
\hline
\end{tabular}

(b)

\begin{tabular}{l}
\hline Staging \\
\hline $\mathrm{H}_{1}$ : impaired systolic or diastolic function of $\mathrm{LV}$ without \\
structural damage \\
$\mathrm{H}_{2}: \mathrm{LV}$ with systolic or diastolic dysfunction and structural \\
damage (hypertrophy previous myocardial infarction) \\
$\mathrm{H}_{3}$ : systolic and diastolic dysfunction (and/or $\left.\mathrm{EF}<35 \%\right)$ with \\
left ventricular remodeling \\
$\mathrm{H}_{4}$ : biventricular systolic and diastolic dysfunction
\end{tabular}

HF and impaired ejection fraction would be incorrect [23]. Pulmonary hypertension, as well as HF, is also an age-related disease [24] and is tightly associated with increasing of left atrial diastolic pressures and increasing in systemic vascular resistances. Consequently, to better establish the severity of HF, the functional evaluation of the pulmonary circulation is necessary. Normal pulmonary arterial pressure has been defined with a value of mPAP (mean pulmonary arterial pressure) $<25 \mathrm{~mm} \mathrm{Hg}$ at rest and $<30 \mathrm{~mm} \mathrm{Hg}$ during exercise, while a normal pulmonary arterial wedge pressure (PAWP) has been defined as $\leq 15 \mathrm{~mm} \mathrm{Hg}$. In order to evaluate PAP, echo-Doppler is largely used, provided that the gold standard to evaluate the overall pulmonary hemodynamic is the right cardiac catheterization [25]. An increase in mPAP value $>$ $25 \mathrm{~mm} \mathrm{Hg}$ with a PAWP value $\leq 15 \mathrm{~mm} \mathrm{Hg}$ is defined as precapillary pulmonary hypertension with negative influence on right ventricle that can lead to cor pulmonale. On the contrary, mPAP > $25 \mathrm{~mm} \mathrm{Hg}$ and PAWP > $15 \mathrm{~mm} \mathrm{Hg}$, define a condition known as postcapillary pulmonary hypertension and it is characteristic of congestive pulmonary disease (Figure 1).

About congestive pulmonary disease, Gheorghiade [26] purposes a fine differentiation between hemodynamic congestion and clinical congestion, endorsing that the first one is a state of volume overload resulting in augmented left ventricular filling pressures, that precedes cardiopulmonary congestion by several days. Clinical congestion, is the combination of cardiopulmonary and systemic signs and symptoms that result from increased left ventricular filling pressures. The congestive state of pulmonary system occurs in HF when the MPAP and the PAWP are increased. It is also possible that this precarious condition lead to acute HF syndrome with pulmonary edema [27].
The early finding of "pulmonary involvement" and their treatment can reduce the progression towards HF. Gheorghiade still supports the usefulness of physical examination to evaluate hemodynamic congestion in the absence of cardiac catheterization. The physical bedside examination includes Valsalva maneuver [28], assessments of orthostatic blood pressure changes, and the heart rate and blood pressure response to sublingual nitroglycerin, in order to identify patients with high left ventricle filling pressures in the absence of signs and symptoms of clinical congestion. Moreover, about lung congestion, also pleural effusion should be considered in decompensated HF $[29,30]$. In Table 2(a), we purpose the parameters that can supply a graduation of pulmonary involvement and in Table 2(b) is shown the Lung Staging in HF.

Obviously, a patient with pulmonary edema will be classified as Clinical Congestion $\mathrm{L}_{2}$. The Cardiac Lung represents the arterialization of precapillary and postcapillary pulmonary vasculature and it is easy to imagine how this condition maximally compromises the respiratory function. In order to offer the best therapy for each patient, pulmonary parameters $\left(\mathrm{L}_{n}\right)$ have to be tightly considered in association with the estimation of cardiac damage expressed by $\mathrm{H}_{n}$. For example, in treating a patient with pulmonary edema, $\mathrm{L}_{2}$ would assume a different role if associated to a cardiac damage estimated as $\mathrm{H}_{1}$ or $\mathrm{H}_{4}$. In fact pulmonary edema could occur in patients with a preserved left ventricular systolic function, but also in patients with severe systolic disfunction (cardiogenic shock) and left ventricular remodeling $\left(\mathrm{H}_{4} \mathrm{~L}_{2}\right)$ or cardiogenic shock, two different conditions with different therapeutic approaches.

2.3. Malfunction of Other Organs. HF brings countless peripheral systemic signs and symptoms and affects practically all the organs. First of all, there is a correlation between renal and cardiac function. Moreover, the incidence of chronic renal failure is increasing as well as that of $\mathrm{HF}$ [31]. Cardiorenal syndromes (CRS) indicate a pathological condition in which there is a tight relationship between cardiovascular system and renal function [32]. CRS is subclassified in five typologies depending on directional relation between kidney and heart. In the type one, a rapid worsening of cardiac function influences the renal function bringing an acute kidney injury (e.g., an acute HF could lead to a cardiogenic renal failure). CRS type two does not result in acute heart damage, but in chronically abnormal heart function that chronically affects the renal function, (e.g., patients with chronic HF often show chronic renal failure). CRS type three is characterized by a sudden worsening of the renal function that leads to acute cardiac injury. CRS type four is represented by a chronic primary renal disease that causes a chronic heart damage overtime. Finally, the CRS type five is characterized by a conjuncted cardiac and renal dysfunctions due to a chronic systemic disease. Figure 2 shows the graphical representation of cardio-renal syndrome and how the two organs can influence one another.

Renal function is an important parameter to consider in order to have an overall assessment of patient with HF. It is 


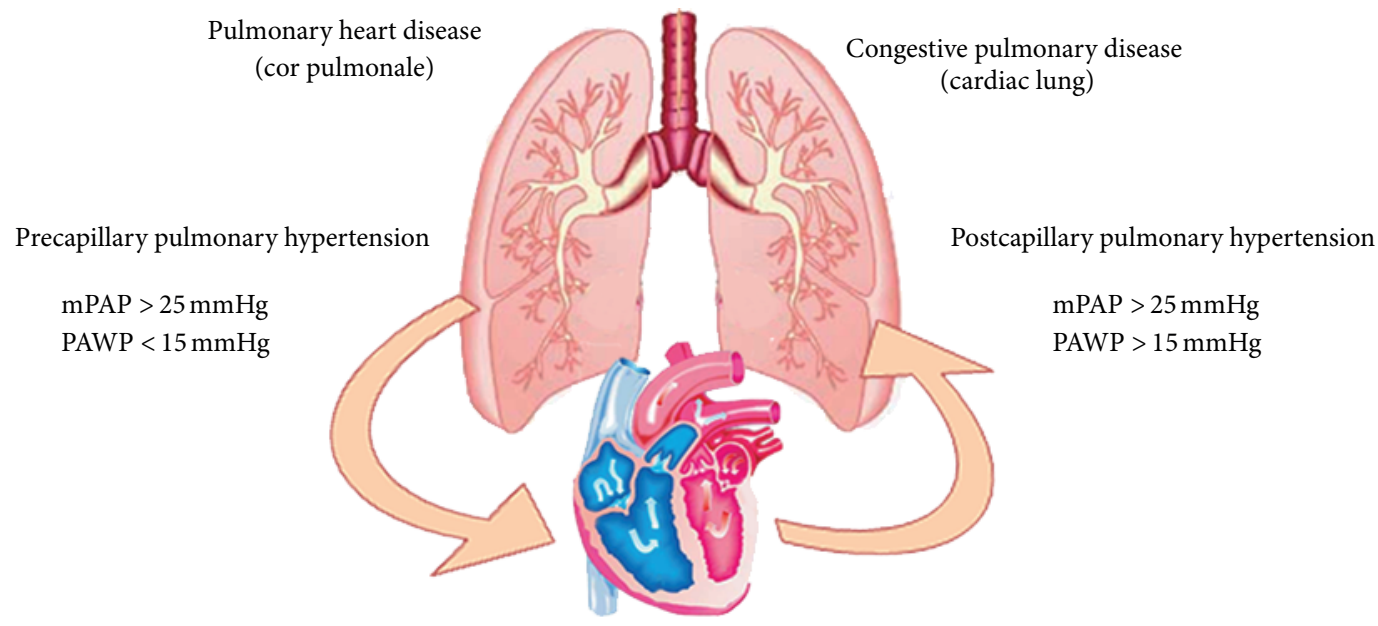

FIGURE 1: Physiopathology and reference range values in precapillary pulmonary hypertension and postcapillary pulmonary hypertension.

TABLE 2: (a) Schematic description of different pulmonary implications occurring in patients with HF. (b) Severity stages of lung involvement.

(a)

\begin{tabular}{l}
\hline Parameters of pulmonary damage \\
\hline Precapillary pulmonary hypertension $(\mathrm{mPAP}>25 \mathrm{mmHg}$ \\
PAWP $<15 \mathrm{mmHg})$ \\
Postcapillary pulmonary hypertension $(\mathrm{mPAP}>25 \mathrm{mmHg}$ \\
PAWP $>15 \mathrm{mmHg})$ \\
Pleural effusion \\
Pulmonary edema
\end{tabular}

(b)

\begin{tabular}{lc}
\hline & Staging \\
\hline $\mathrm{L}_{1}$ & Hemodynamic congestion \\
$\mathrm{L}_{2}$ & Clinical congestion \\
$\mathrm{L}_{3}$ & Cardiac lung \\
\hline
\end{tabular}

possible to investigate some parameters in order to define renal function and its impairment grade.

Creatinine $(0.7$ to $1.3 \mathrm{VN} \mathrm{mg/dL})$ alone is not a reliable parameter for the measurement of renal function. Indeed, as produced by the muscles and eliminated by the kidney, it increases with muscle mass. Therefore, a value of $1.2 \mathrm{mg} / \mathrm{dL}$ is a normal expression of renal function in a patient with muscle mass particularly developed; on the contrary, the same value can "mask" a frank renal failure in a patient with low muscle mass. Furthermore, especially in the early stages of renal failure, small increases in the serum creatinine value indicate significant decreases in glomerular filtration rate (GFR). For these reasons, assessment of renal function cannot only be based on serum creatinine, but it is necessary to determine the GFR: the lower the creatinine clearance is, the lower the patients prognosis is [33]. In this context the evaluation of renal function, by calculating creatinine clearance [34], provides an optimal parameter to estimate peripheral signs of HF. The two main formulas for the calculation of GFR

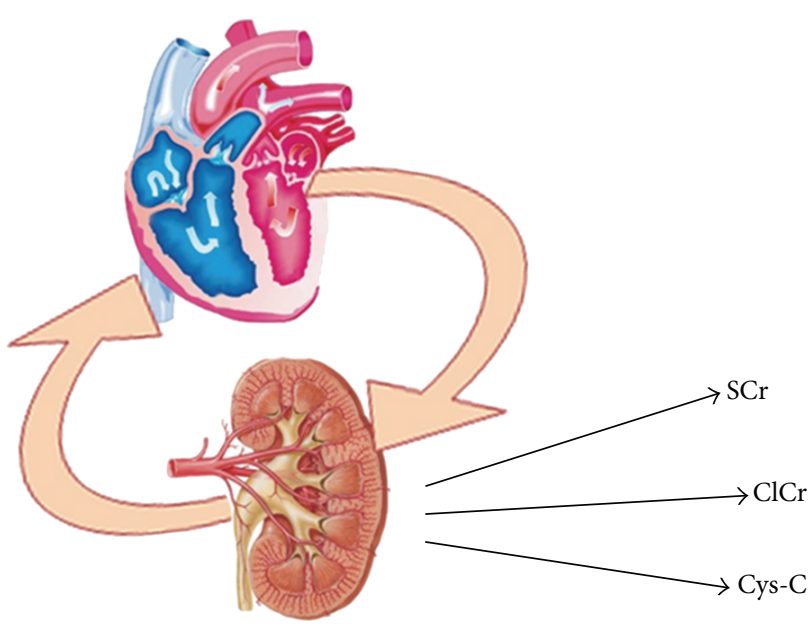

FIGURE 2: The concept of cardiorenal syndrome that often brings the insaturation of a vicious circle is graphically explicated. SCr: Serum Creatinine; ClCr: Clearance of Creatinine; Cys-C: Cystatine-C.

are the Cockroft-Gault and MDRD (Modification of Diet in Renal Disease), although currently the most widely used is a modification of the simplified MDRD formula (sMDRD or simplified MDRD).

Creatinine clearance, calculated by Cockroft-Gault formula, takes in consideration creatinine serum concentration (mg/dL), age (in years), weight (in Kg), and gender (multiplying the total score for 0.85 in female patients). The complete formula is $[(140-$ age $) \times$ weight $] / 72 \times$ serum creatinine concentration; the value resulted is multiplied by 0.85 in female patients. The reference values are 57-115 in women and 95-145 in men [35].

Simplified MDRD formula, to calculate creatinine clearance, takes in consideration parameters as serum creatinine, ethnicity, and gender. The formula is GFR ( $\mathrm{mL} / \mathrm{min} /$ $\left.1.73 \mathrm{~m}^{2}\right)=186 \times$ serum creatinine $-1.154(\mu \mathrm{mol} / \mathrm{L}) \times$ age $0.203(\times 0.742$ if the patient is a woman $)(\times 1.21$ if the patient is black) [36]. Moreover, cystatin C (Cys-c), an endogenous 
marker of glomerular filtration rate (GFR), is another useful parameter to assess renal function also in patients with $\mathrm{HF}$ [37]. Cys-C is a part of the cysteine protease and its serum concentration increases when the GFR diminishes. Since creatinine concentration is influenced by muscle mass and growth and also by other pathological conditions as liver cirrhosis, anorexia nervosa, and so on, Cys- $\mathrm{C}$ has proved to be a more reliable parameter in assessing renal function [38]. The usefulness of Cys- $\mathrm{C}$ in $\mathrm{HF}$ has recently been confirmed by the study of Campbell et al. that demonstrated a poor outcome in patients with high level of Cys- $\mathrm{C}$ and impaired renal function [39].

It is possible to evaluate blood urea nitrogen (BUN) in order to complete the renal assessment, but it is necessary to consider that BUN is an ambiguous marker of renal impairment because it is strongly influenced by catabolism and catabolic alteration. The parameters to be considered for the evaluation of renal function are shown in Table 3(a).

In order to overview other possible damaged organs in $\mathrm{HF}$, we report an interesting study that supports this concept: "heart disease affecting the liver and liver disease affecting the heart" [40]. HF brings liver complications like alterations of functional liver tests that recover to normal values with the compensation of HF. The study of Allen et al. brings forth that an increment in total bilirubin, alkaline phosphatase, and ALT (alanine transaminase), and a reduction in albumin, are reliable in patients with HF [41]. Moreover, the study proves that this kind of data variably influences the prognosis of patients with HF. To assess liver dysfunction some scores were structured, in particular Child-Pugh score and MELD (Model for End Stage Liver Disease) score. MELD score takes into account total bilirubin, serum creatinine, and INR; Child-Pugh evaluates total bilirubin, serum albumin, INR, ascites, and hepatic encephalopathy. Notice how in both scores created to evaluate liver function, INR and total bilirubin are considered [42]. The INR should be considered carefully since about $1 / 3$ of HF patients is on anticoagulant therapy.

The etiology of liver congestion in HF depends on different variables [43]. When the pulmonary vascular resistance increases and the mPAP is over $25 \mathrm{~mm} \mathrm{Hg}$, the repercussion of high pressure induces a volume overload in portal circulation. It is well known that right HF causes liver congestion. For this clinical evidence, in staging HF, it is necessary to consider the hemodynamic and organic function of liver. To obtain an estimate of hemodynamic changes in hepatic district, we can measure the main portal vein flow velocity using duplex sonography $[44,45]$. A reduced portal flow that can be detected is likely a consequence of HF. The increment of portal pressure due to right HF could remain silent for years before clinical manifestations occurrence. Another very important parameter that physicians may consider to estimate the congestion state of venous system is the collapse of the inferior vena cava measured with ultrasonography during maximum inspiration. In this regard, a study published by Blehar and coworkers, shows how the diameter variations of the inferior vena cava are related to volume overload in patients with HF [46]. Analogous to the events that occur in kidneys, what occurs in liver during HF ("liver impairment")
TABle 3: (a) Parameters of kidney damage. (b) Hepatic damage parameters.

(a)

\begin{tabular}{l}
\hline Parameters of kidney damage \\
\hline Glomerular filtration rate \\
Blood urea nitrogen \\
Serum creatinine \\
Clearance of creatinine \\
Cystatin C
\end{tabular}

(b)

\begin{tabular}{l}
\hline Parameters of hepatic damage \\
\hline -GT \\
Bilirubine (total) increased \\
ALT, AST \\
Alkaline phosphatase increased \\
Albumin decreased \\
Diminished INR value \\
Ascites \\
Impairment of portal blood flow \\
No changes of inferior vena cava diameter variation during \\
inspiration
\end{tabular}

is to be considered as a consequence of a more advanced disease. We must underline that, to give a careful estimate of liver damage in HF, it is necessary to evaluate all the parameters summarized in Figure 3 and Table 3(b). In fact, the staging of liver damage based on only one of these parameters is simplistic and misleading because it is known that liver function tests are aspecific indices of deterioration of liver and are therefore to be interpreted in order to make a differential diagnosis with other hepatic diseases. During the investigation of liver's impairment, we can face other clinical features as ascites. Cardiac ascites might indeed develop in patients with a right ventricular failure and systemic venous hypertension [47].

Finally, another organ that can be affected by HF is also central nervous system. In fact cerebral hypoperfusion and impaired cerebral function occur in $\operatorname{HF}[48,49]$. Cerebral vasoreactivity depends on cardiac functional variables. Cerebral complication that can occur in HF is also another consequence of progression of cardiac disease. Other important aspects of brain involvement in HF are psychological troubles. We mainly refer to the depression. In this regard, the research reported by Gottlieb et al. is very interesting [50]. This study demonstrated that a great part of enrolled patients with HF showed signs of depression and that the coexisting depression severely impacts on life's quality and prognosis. Finally, in a comprehensive assessment of the patient with $\mathrm{HF}$ it is important to remember a parameter that gives us an idea of the cachectic state of the subject and is representative of multiple organ failure. The most robust and studied marker that can do this is the BMI (mass $(\mathrm{Kg}) /$ height $\left.(\mathrm{m})^{2}\right)[51,52]$. 


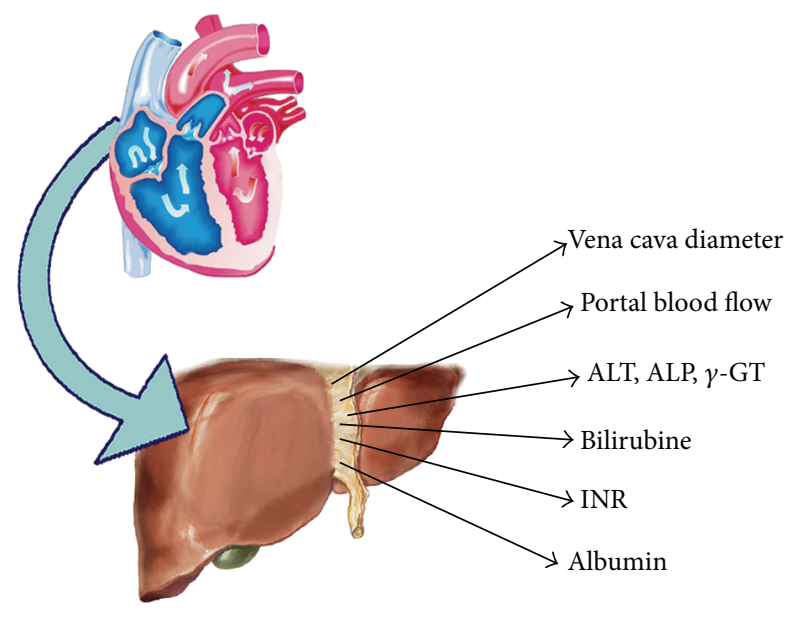

FIgURE 3: Shows the way of liver's impairment in HF. ALT: Alanine Transaminase; ALP: Alkaline Phosphatase; INR: International Normalized Ratio.

In conclusion, we would like to purpose this score to weight organ's malfunction that can occur in HF. Considering the presence and the number of malfunctioning organs in HF, we can have the $M$ staging as represented in Table 4 . The staging of HF influences the prognosis of patients more than their therapy, but, without any doubt, it is necessary to take into consideration any single "malfunction of other organs" in order to guarantee the best treatments and the best quality of life in patients with HF.

\section{Conclusion}

In our opinion it can be very useful to gain a classification of $\mathrm{HF}$ and its progression with a methodological scoring like the TNM evaluation used in oncology. The latest guidelines are mostly based on syndromic and symptomatic classification of HF that, in some cases, could be insufficient. Moreover, the NYHA classification has already been shown to be insubstantial in the evaluation of some kind of patients [53, 54]. We support this viewpoint because this symptomatic classification cannot give a real overview of general health state of the patients with HF. The progression of clinical research in the ambit of HF's classification is still open. What has been written in these pages is intended to provide a classification to proportion our interventions depending on the type of patients we face and to better consider their degree of disease.

With a TNM-like evaluation we do not risk to rely on single parameters, sometimes aspecific, but we have the opportunity to evaluate all the necessary parameters and enclose them in a single compressive classification representing the patient in that moment. This classification will allow us to use a traditional therapy for patients in initial stage of HF $\left(\mathrm{H}_{1} \mathrm{~L}_{1} \mathrm{M}_{0}\right)$ and to apply second-tier line therapies in patients with $\mathrm{H}_{2} \mathrm{~L}_{2} \mathrm{M}_{n}$ stage. In fact, the presence of malfunction of other organs authorizes the physicians to employ type of therapies that will be not only cardioprotective, but also nephroprotective, hepatoprotective, and so on, justifying
TABLE 4: Staging of malfunction of other organs.

\begin{tabular}{ll}
\hline Score & Staging \\
\hline 0 & $\mathrm{M}_{0}:$ no malfunction of other organs \\
1 & $\mathrm{M}_{1}:$ single organ damage due to $\mathrm{HF}$ \\
2 & $\mathrm{M}_{2}:$ double organ damage due to $\mathrm{HF}$ \\
$\geq 3$ & $\mathrm{M}_{3}:$ multiple organ damage \\
\hline
\end{tabular}

the increased cost of therapy in proportion to the benefits obtained. Besides, this type of classification can be very useful in assessing the true end-stage patients $\left(\mathrm{H}_{4} \mathrm{~L}_{3} \mathrm{M}_{3}\right)$. In these cases, the classification HLM allows us to pursue the most appropriate therapy targeting the quality of life remained with palliative support.

Starting with a patients' followup, applying this new staging system, the future prospect is to stratify prognosis, consider the course of therapy, and evaluate cardiovascular events of patients affected by HF.

\section{References}

[1] S. Neubauer, "The failing heart-an engine out of fuel," New England Journal of Medicine, vol. 356, no. 11, pp. 1140-1151, 2007.

[2] H. Krum and W. T. Abraham, "Heart failure," The Lancet, vol. 373, no. 9667, pp. 941-955, 2009.

[3] M. Gheorghiade and P. S. Pang, "Acute heart failure syndromes," Journal of the American College of Cardiology, vol. 53, no. 7, pp. 557-573, 2009.

[4] S. Stewart, K. MacIntyre, S. Capewell, and J. J. V. McMurray, "Heart failure and the aging population: an increasing burden in the 21st century?" Heart, vol. 89, no. 1, pp. 49-53, 2003.

[5] S. A. Hunt, W. T. Abraham, M. H. Chin et al., "2009 focused update incorporated into the ACC/AHA 2005 guidelines for the diagnosis and management of heart failure in adults. A Report of the American College of Cardiology Foundation/American Heart Association Task Force on Practice Guidelines Developed in Collaboration With the International Society for Heart and Lung Transplantation," Journal of the American College of Cardiology, vol. 53, no. 15, pp. el-e90, 2009.

[6] M. Senni, G. Santilli, P. Parrella et al., "A novel prognostic index to determine the impact of cardiac conditions and comorbidities on one-year outcome in patients with heart failure," American Journal of Cardiology, vol. 98, no. 8, pp. 1076-1082, 2006.

[7] I. Cygankiewicz, W. Zareba, R. Vazquez et al., "Risk stratification of mortality in patients with heart failure and left ventricular ejection fraction >35\%.," American Journal of Cardiology, vol. 103, no. 7, pp. 1003-1010, 2009.

[8] R. Vazquez, A. Bayes-Genis, I. Cygankiewicz et al., "The MUSIC Risk score: a simple method for predicting mortality in ambulatory patients with chronic heart failure," European Heart Journal, vol. 30, no. 9, pp. 1088-1096, 2009.

[9] A. P. Kalogeropoulos, V. V. Georgiopoulou, G. Giamouzis et al., "Utility of the Seattle Heart Failure Model in patients with advanced heart failure," Journal of the American College of Cardiology, vol. 53, no. 4, pp. 334-342, 2009.

[10] P. N. Peterson, J. S. Rumsfeld, L. Liang et al., "A validated risk score for in-hospital mortality in patients with heart failure from the American heart association get with the guidelines program," Circulation, vol. 3, no. 1, pp. 25-32, 2010. 
[11] P. N. Peterson, J. S. Rumsfeld, L. Liang et al., "Treatment and risk in heart failure gaps in evidence or quality?" Circulation, vol. 3 , no. 3, pp. 309-315, 2010.

[12] R. J. Epstein, “TNM: therapeutically not mandatory," European Journal of Cancer, vol. 45, no. 7, pp. 1111-1116, 2009.

[13] G. Ligabue, F. Fiocchi, S. Ferraresi, A. Barbieri, R. Romagnoli, and P. Torricelli, "How to quantify infarct size on delayedenhancement MR images: a comparison between visual and quantitative approach," Radiologia Medica, vol. 112, no. 7, pp. 959-968, 2007.

[14] T. Baks, F. Cademartiri, A. D. Moelker et al., "Multislice computed tomography and magnetic resonance imaging for the assessment of reperfused acute myocardial infarction," Journal of the American College of Cardiology, vol. 48, no. 1, pp. 144-152, 2006.

[15] A. Seixas Bello Moreira, "Left ventricular hypertrophy induced by overnutrition early in life," Nutrition, Metabolism and Cardiovascular Diseases, vol. 19, pp. 805-810, 2009.

[16] B. J. Maron, "Hypertrophic cardiomyopathy: a systematic review," Journal of the American Medical Association, vol. 287, no. 10, pp. 1308-1320, 2002.

[17] S. Patanè, "Revelation of an obstructive hypertrophic cardiomyopathy in an elderly patient," International Journal of Cardiology, vol. 145, pp. e41-e43, 2009.

[18] K. S. Dujardin, M. Enriquez-Sarano, A. Rossi, K. R. Bailey, and J. B. Seward, "Echocardiographic assessment of left ventricular remodeling: are left ventricular diameters suitable tools?" Journal of the American College of Cardiology, vol. 30, pp. 1534-1541, 1997.

[19] R. V. Shah and M. J. Semigran, "Pulmonary hypertension secondary to left ventricular systolic dysfunction: contemporary diagnosis and management," Current Heart Failure Reports, vol. 5, no. 4, pp. 226-232, 2008.

[20] A. Uzunpinar and M. Cilingiroglu, "Pulmonary arterial hypertension," Current Atherosclerosis Reports, vol. 11, no. 2, pp. 139145, 2009.

[21] D. Bonderman, A. M. Martischnig, D. Moertl, and I. M. Lang, "Pulmonary hypertension in chronic heart failure," International Journal of Clinical Practice, vol. 63, no. 161, supplement, pp. 4-10, 2009.

[22] C. S. P. Lam, V. L. Roger, R. J. Rodeheffer, B. A. Borlaug, F. T. Enders, and M. M. Redfield, "Pulmonary hypertension in heart failure with preserved ejection fraction. A community-based study," Journal of the American College of Cardiology, vol. 53, no. 13, pp. 1119-1126, 2009.

[23] W. C. Little, "Heart failure with a normal left ventricular ejection fraction: diastolic heart failure," Transactions of the American Clinical and Climatological Association, vol. 119, pp. 93-102, 2008.

[24] C. S. P. Lam, B. A. Borlaug, G. C. Kane, F. T. Enders, R. J. Rodeheffer, and M. M. Redfield, "Age-associated increases in pulmonary artery systolic pressure in the general population," Circulation, vol. 119, no. 20, pp. 2663-2670, 2009.

[25] D. B. Badesch, H. C. Champion, M. A. Gomez Sanchez et al., "Diagnosis and assessment of pulmonary arterial hypertension," Journal of the American College of Cardiology, vol. 54, no. 1, supplement, pp. S55-S66, 2009.

[26] M. Gheorghiade, G. Filippatos, L. De Luca, and J. Burnett, "Congestion in acute heart failure syndromes: an essential target of evaluation and treatment," American Journal of Medicine, vol. 119, no. 12, pp. S3-S10, 2006.
[27] R. M. Effros and J. C. Parker, "Pulmonary vascular heterogeneity and the Starling hypothesis," Microvascular Research, vol. 78, no. 1, pp. 71-77, 2009.

[28] G. M. Felker, P. S. Cuculich, and M. Gheorghiade, "The Valsalva maneuver: a bedside "biomarker" for heart failure," American Journal of Medicine, vol. 119, no. 2, pp. 117-122, 2006.

[29] H. Kataoka, "Pericardial and pleural effusions in decompensated chronic heart failure," American Heart Journal, vol. 139, no. 5, pp. 918-923, 2000.

[30] I. Gotsman, Z. Fridlender, A. Meirovitz, D. Dratva, and M. Muszkat, "The evaluation of pleural effusions in patients with heart failure," American Journal of Medicine, vol. 111, no. 5, pp. 375-378, 2001.

[31] L. G. Bongartz, M. J. Cramer, P. A. Doevendans, J. A. Joles, and B. Braam, "The severe cardiorenal syndrome: "Guyton revisited'”' European Heart Journal, vol. 26, no. 1, pp. 11-17, 2005.

[32] C. Ronco, M. Haapio, A. A. House, N. Anavekar, and R. Bellomo, "Cardiorenal syndrome," Journal of the American College of Cardiology, vol. 52, no. 19, pp. 1527-1539, 2008.

[33] J. R. González-Juanatey, "The question of heart failure in ONTARGET and TRANSCEND: implications for clinical practice," Journal of Hypertension, vol. 27, no. 2, supplement, pp. S32S35, 2009.

[34] G. Francis, "Acute decompensated heart failure: the cardiorenal syndrome," Cleveland Clinic Journal of Medicine, vol. 73, supplement 2, pp. S8-S30, 2006.

[35] D. W. Cockcroft and M. H. Gault, "Prediction of creatinine clearance from serum creatinine," Nephron, vol. 16, no. 1, pp. 3141, 1976.

[36] N. Lameire, A. Adam, C. R. Becker et al., "Baseline renal function screening," American Journal of Cardiology, vol. 98, no. 6, pp. 21-26, 2006.

[37] A. Moran, R. Katz, N. L. Smith et al., "Cystatin C concentration as a predictor of systolic and diastolic heart failure," Journal of Cardiac Failure, vol. 14, no. 1, pp. 19-26, 2008.

[38] G. Filler, A. Bökenkamp, W. Hofmann, T. Le Bricon, C. Martínez-Brú, and A. Grubb, "Cystatin C as a marker of GFRhistory, indications, and future research," Clinical Biochemistry, vol. 38, no. 1, pp. 1-8, 2005.

[39] C. Y. Campbell, W. Clarke, H. Park, N. Haq, B. B. Barone, and D. J. Brotman, "Usefulness of cystatin C and prognosis following admission for acute heart failure," American Journal of Cardiology, vol. 104, no. 3, pp. 389-392, 2009.

[40] J. E. Naschitz, G. Slobodin, R. J. Lewis, E. Zuckerman, and D. Yeshurun, "Heart diseases affecting the liver and liver diseases affecting the heart," American Heart Journal, vol. 140, no. 1, pp. 111-120, 2000.

[41] L. A. Allen, G. M. Felker, S. Pocock et al., "Liver function abnormalities and outcome in patients with chronic heart failure: data from the Candesartan in Heart Failure. Assessment of Reduction in Mortality and Morbidity (CHARM) program," European Journal of Heart Failure, vol. 11, no. 2, pp. 170-177, 2009.

[42] T.-I. Huo, J.-C. Wu, H.-C. Lin et al., "Evaluation of the increase in model for end-stage liver disease (DMELD) score over time as a prognostic predictor in patients with advanced cirrhosis: risk factor analysis and comparison with initial MELD and Child-Turcotte-Pugh score," Journal of Hepatology, vol. 42, no. 6, pp. 826-832, 2005.

[43] M. Porres-Aguilar, M. J. Zuckerman, J. B. Figueroa-Casas, and M. J. Krowka, "Portopulmonary hypertension: state of the art," Annals of Hepatology, vol. 7, no. 4, pp. 321-330, 2008. 
[44] S.-S. Yang, "Duplex Doppler ultrasonography in portal hypertension," Journal of Medical Ultrasound, vol. 15, no. 2, pp. 103111, 2007.

[45] L. Zhang, J. Yin, Y. Duan, Y. Yang, L. Yuan, and T. Cao, "Assessment of intrahepatic blood flow by Doppler ultrasonography: relationship between the hepatic vein, portal vein, hepatic artery and portal pressure measured intraoperatively in patients with portal hypertension," BMC Gastroenterology, vol. 11, article 84, 2011.

[46] D. J. Blehar, E. Dickman, and R. Gaspari, "Identification of congestive heart failure via respiratory variation of inferior vena cava diameter," American Journal of Emergency Medicine, vol. 27, no. 1, pp. 71-75, 2009.

[47] C. C. Giallourakis, P. M. Rosenberg, and L. S. Friedman, "The liver in heart failure," Clinics in Liver Disease, vol. 6, no. 4, pp. 947-967, 2002.

[48] D. Georgiadis, M. Sievert, S. Cencetti et al., "Cerebrovascular reactivity is impaired in patients with cardiac failure," European Heart Journal, vol. 21, no. 5, pp. 407-413, 2000.

[49] C. J. Mathias, "Cerebral hypoperfusion and impaired cerebral function in cardiac failure," European Heart Journal, vol. 21, no. 5, p. 346, 2000.

[50] S. S. Gottlieb, M. Khatta, E. Friedmann et al., "The influence of age, gender, and race on the prevalence of depression in heart failure patients," Journal of the American College of Cardiology, vol. 43, no. 9, pp. 1542-1549, 2004.

[51] A. J. Burger, Y. Han, and D. Aronson, “The relationship between body mass index and mortality in patients with acute decompensated heart failure," International Journal of Cardiology, vol. 134, no. 1, pp. 132-135, 2009.

[52] E. Colín-Ramírez, A. Orea-Tejeda, and L. Castillo-Martínez, "Malnutrition syndrome, but not body mass index, is associated to worse prognosis in heart failure patients Original Research Article," Clinical Nutrition, 2011.

[53] N. Koren-Morag, U. Goldbourt, and D. Tanne, "Poor functional status based on the New York Heart Association classification exposes the coronary patient to an elevated risk of ischemic stroke," American Heart Journal, vol. 155, no. 3, pp. 515-520, 2008.

[54] P. Moons, K. Van Deyk, and W. Budts, “The NYHA classification, employment, and physical activities are poor indicators of quality of life after congenital cardiac surgery," Annals of Thoracic Surgery, vol. 82, no. 3, pp. 1167-1168, 2006. 


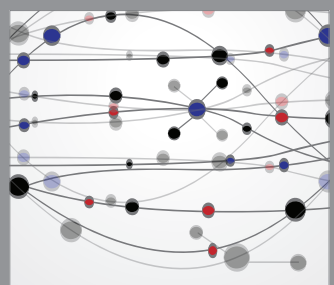

The Scientific World Journal
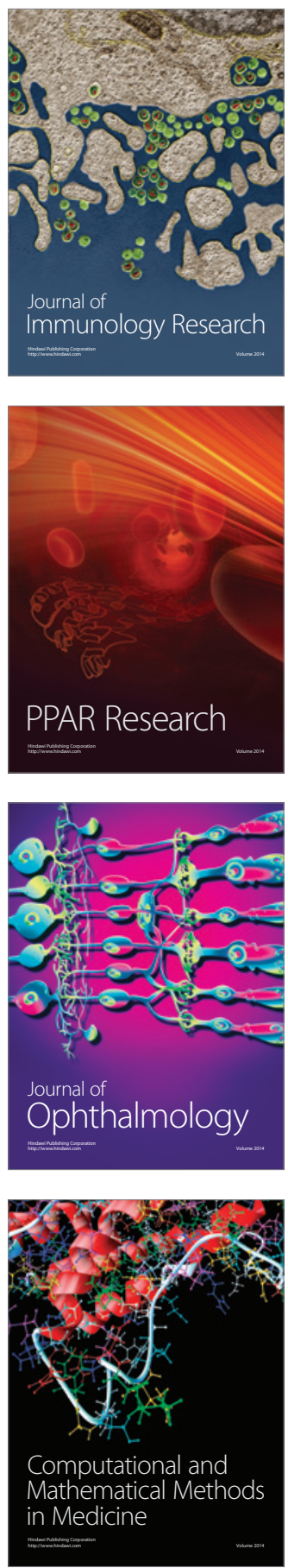

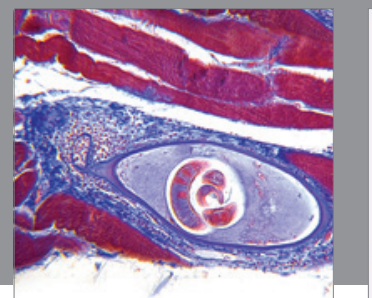

Gastroenterology

Research and Practice
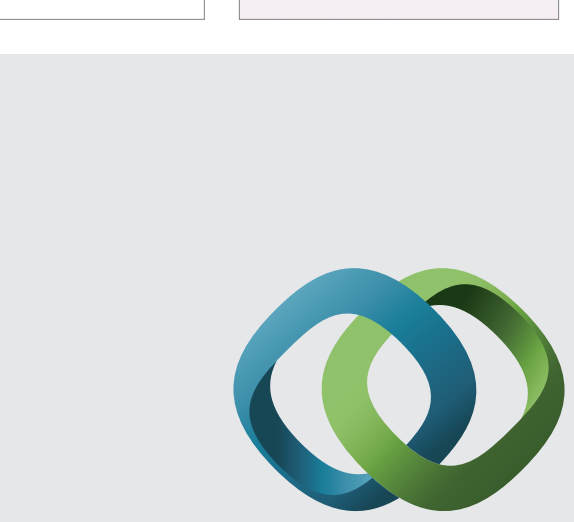

\section{Hindawi}

Submit your manuscripts at

http://www.hindawi.com
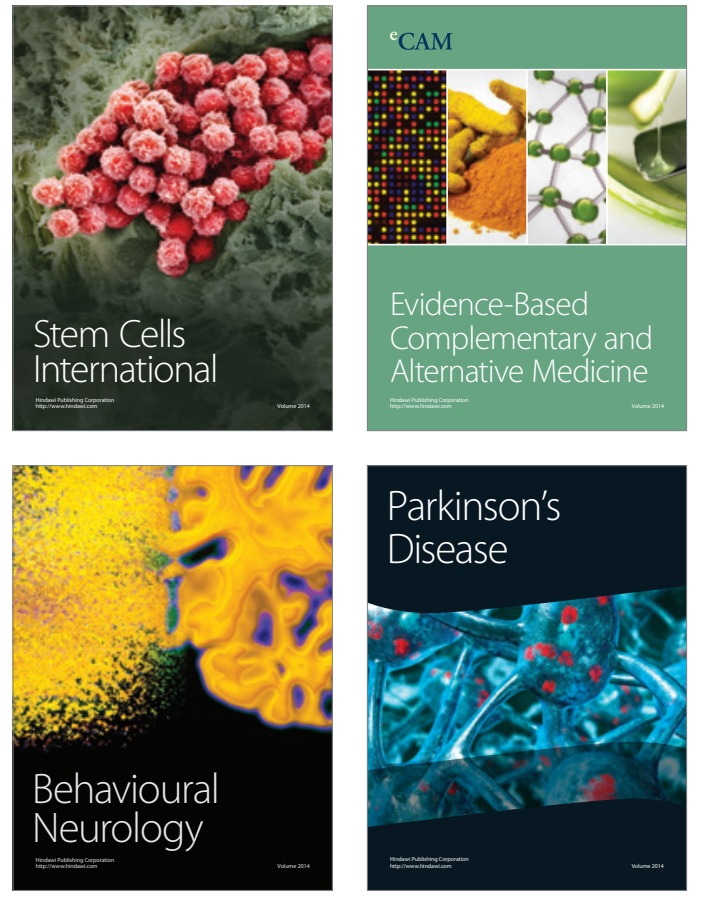
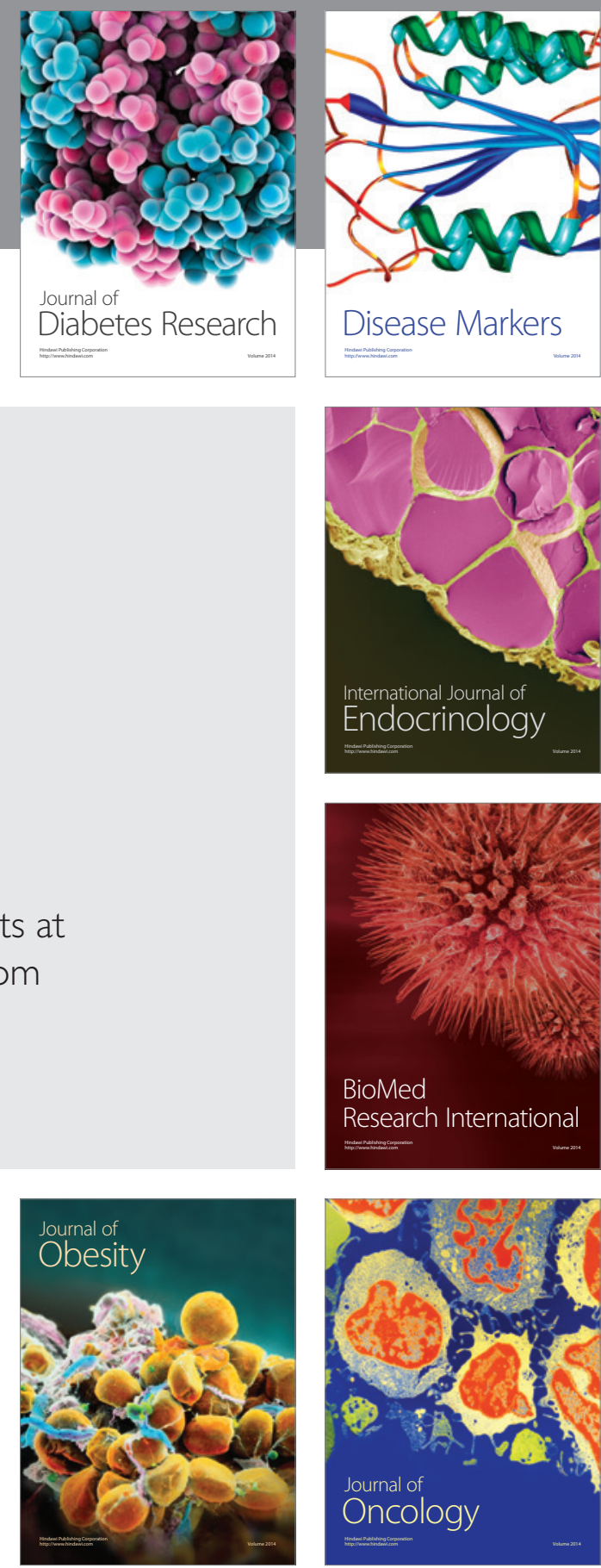

Disease Markers
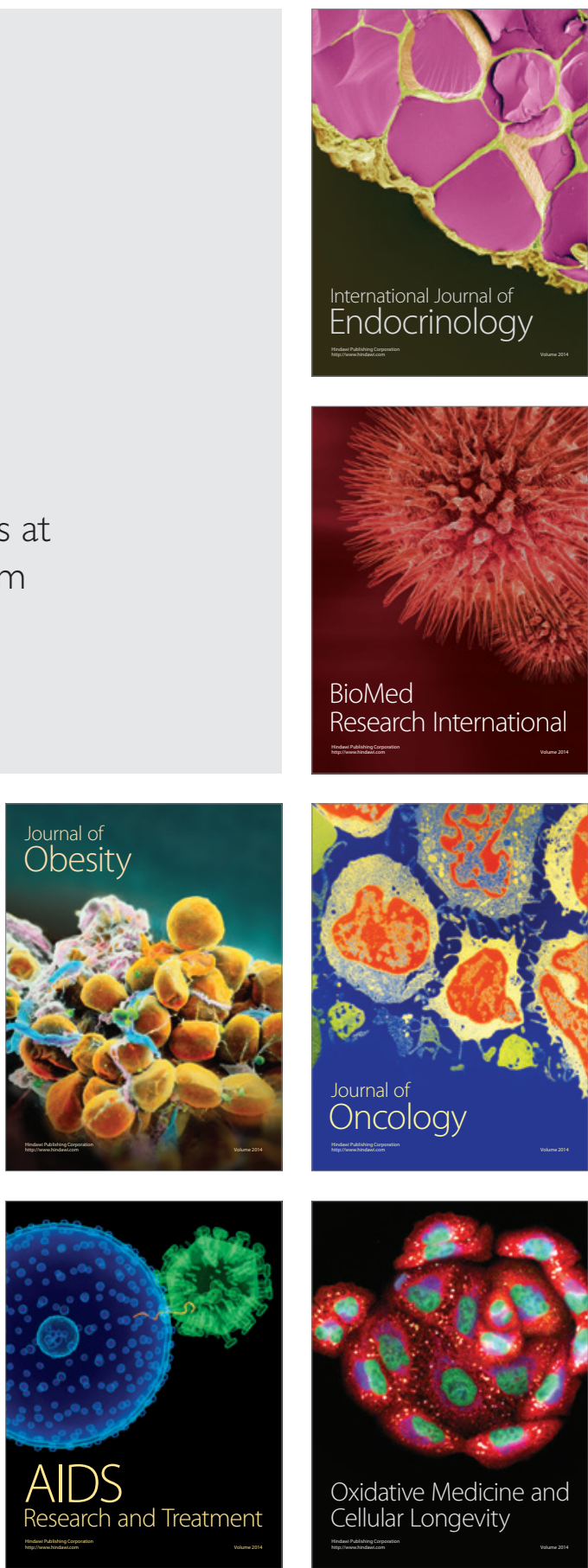